\title{
Chronic Maxillary Sinusitis
}

National Cancer Institute

\section{Source}

National Cancer Institute. Chronic Maxillary Sinusitis. NCI Thesaurus. Code C34477.

Inflammation of the maxillary sinus that typically lasts beyond eight weeks. It is caused by infections, allergies, and the presence of sinus polyps or a deviated septum. Signs and symptoms include headache, nasal discharge, swelling in the face, dizziness, and breathing difficulties. 